\title{
Erratum: Systematic study of one-loop Dirac neutrino masses and viable dark matter candidates [Phys. Rev. D 96, 095004 (2017)]
}

\author{
Chang-Yuan Yao and Gui-Jun Ding
}

(Received 25 June 2018; published 2 August 2018)

DOI: 10.1103/PhysRevD.98.039901

In the original paper, we made a mistake and some of the models we proposed cannot explain Dirac neutrino masses. The reason is that the mediator particles of some models are electrically charged, which cannot be coupled with the neutral

TABLE VIII. The finite diagrams generated from the topology T8. We list the possible quantum number assignments for the mediators, the expressions for the neutrino mass matrix, and the dark matter candidates. We also display the values of $\alpha$ excluded from the appearance of tree level and Ma diagrams, where $\varnothing$ and $\mathbb{U}$ denote empty set and universal set respectively.

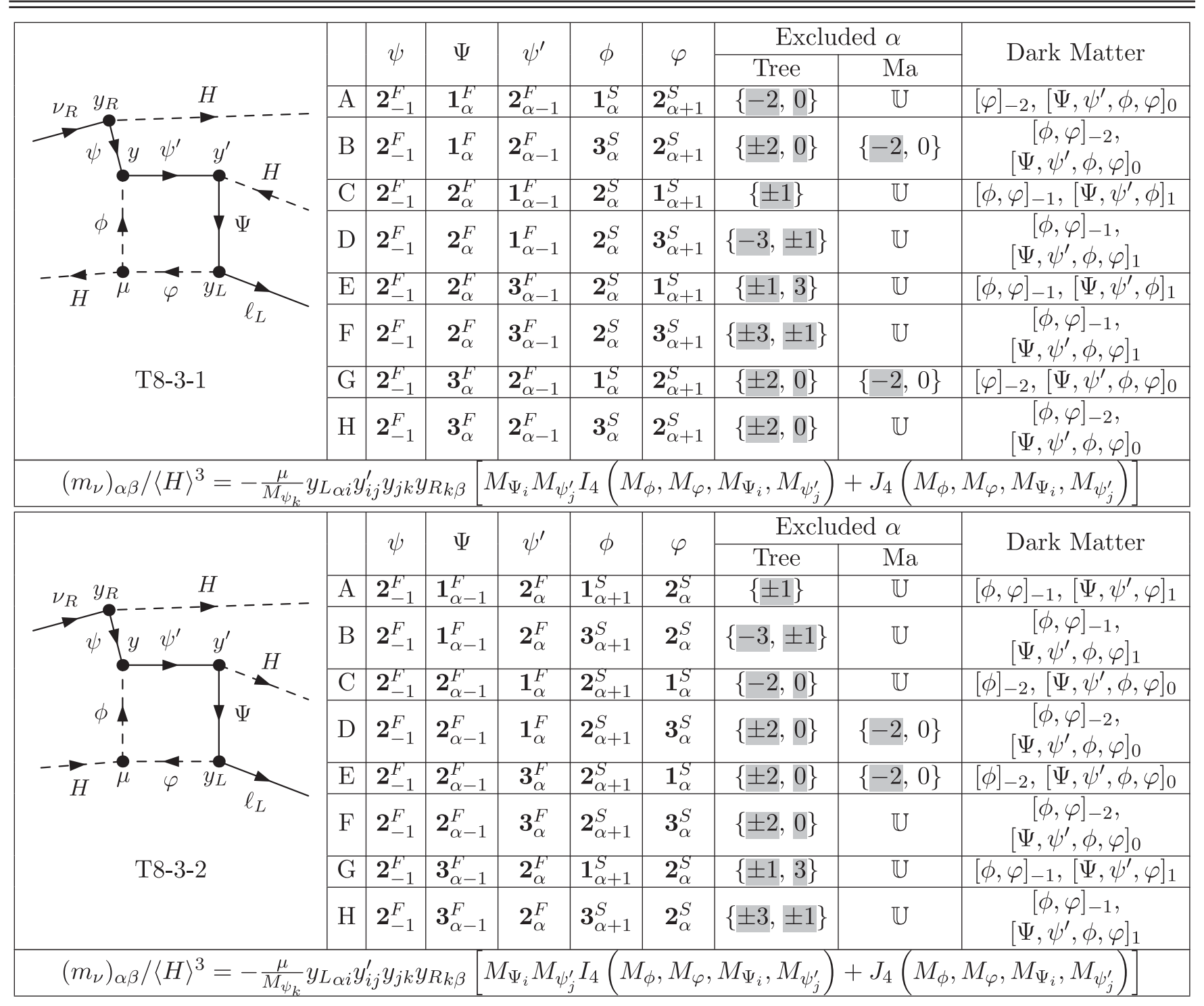

(Table continued) 


\begin{tabular}{|c|c|c|c|c|c|c|c|c|c|}
\hline \multirow{10}{*}{ 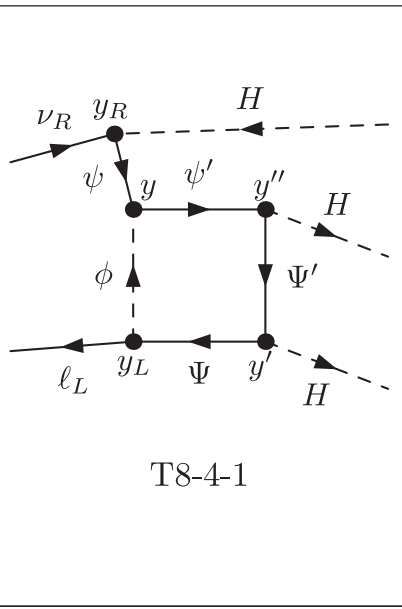 } & & \multirow{2}{*}{$\psi$} & \multirow{2}{*}{$\Psi$} & \multirow{2}{*}{$\psi^{\prime}$} & \multirow{2}{*}{$\Psi^{\prime}$} & \multirow{2}{*}{$\phi$} & \multicolumn{2}{|c|}{ Excluded $\alpha$} & \multirow{2}{*}{ Dark Matter } \\
\hline & & & & & & & Tree & $\mathrm{Ma}$ & \\
\hline & A & $\mathbf{2}_{1}^{F}$ & $\mathbf{1}_{\alpha-1}^{F}$ & $\mathbf{1}_{\alpha+1}^{F}$ & $\mathbf{2}_{\alpha}^{F}$ & $\mathbf{2}_{\alpha}^{S}$ & $\{ \pm 1\}$ & $\mathbb{U}$ & $\begin{array}{c}{\left[\psi^{\prime}, \Psi^{\prime}, \phi\right]_{-1}} \\
{\left[\Psi, \Psi^{\prime}, \phi\right]_{1}}\end{array}$ \\
\hline & B & $\mathbf{2}_{1}^{F}$ & $\mathbf{1}_{\alpha-1}^{F}$ & $\mathbf{3}_{\alpha+1}^{F}$ & $\mathbf{2}_{\alpha}^{F}$ & $2_{\alpha}^{S}$ & $\{-3, \pm 1\}$ & $\mathbb{U}$ & $\begin{array}{l}{\left[\psi^{\prime}, \Psi^{\prime}, \phi\right]_{-1}} \\
{\left[\Psi, \psi^{\prime}, \Psi^{\prime}, \phi\right]_{1}}\end{array}$ \\
\hline & $\mathrm{C}$ & $\mathbf{2}_{1}^{F}$ & $\mathbf{2}_{\alpha-1}^{F}$ & $\mathbf{2}_{\alpha+1}^{F}$ & $\mathbf{1}_{\alpha}^{F}$ & $\mathbf{1}_{\alpha}^{S}$ & $\{0\}$ & $\mathbb{U}$ & {$\left[\Psi, \psi^{\prime}, \Psi^{\prime}, \phi\right]_{0}^{\diamond}$} \\
\hline & $\mathrm{D}$ & $\mathbf{2}_{1}^{F}$ & $\mathbf{2}_{\alpha-1}^{F}$ & $\mathbf{2}_{\alpha+1}^{F}$ & $\mathbf{1}_{\alpha}^{F}$ & $\mathbf{3}_{\alpha}^{S}$ & $\{ \pm 2,0\}$ & $\varnothing$ & {$\left[\Psi, \psi^{\prime}, \Psi^{\prime}, \phi\right]_{0}^{\diamond}$} \\
\hline & $\mathrm{E}$ & $\mathbf{2}_{1}^{F}$ & $\mathbf{2}_{\alpha-1}^{F}$ & $\mathbf{2}_{\alpha+1}^{F}$ & $3_{\alpha}^{F}$ & $\mathbf{1}_{\alpha}^{S}$ & $\{ \pm 2,0\}$ & $\varnothing$ & {$\left[\Psi, \psi^{\prime}, \Psi^{\prime}, \phi\right]_{0}^{\diamond}$} \\
\hline & $\mathrm{F}$ & $2_{1}^{F}$ & $\mathbf{2}_{\alpha-1}^{F}$ & $\mathbf{2}_{\alpha+1}^{F}$ & $3_{\alpha}^{F}$ & $3_{\alpha}^{S}$ & $\{ \pm 2,0\}$ & $\mathbb{U}$ & {$\left[\Psi, \psi^{\prime}, \Psi^{\prime}, \phi\right]_{0}^{\diamond}$} \\
\hline & $\mathrm{G}$ & $\mathbf{2}_{1}^{F}$ & $\mathbf{3}_{\alpha-1}^{F}$ & $\mathbf{1}_{\alpha+1}^{F}$ & $\mathbf{2}_{\alpha}^{F}$ & $2_{\alpha}^{S}$ & $\{ \pm 1,3\}$ & $\mathbb{U}$ & $\begin{array}{c}{\left[\Psi, \psi^{\prime}, \Psi^{\prime}, \phi\right]_{-1}} \\
{\left[\Psi, \Psi^{\prime}, \phi\right]_{1}}\end{array}$ \\
\hline & $\mathrm{H}$ & $\mathbf{2}_{1}^{F}$ & $\mathbf{3}_{\alpha-1}^{F}$ & $\mathbf{3}_{\alpha+1}^{F}$ & $\mathbf{2}_{\alpha}^{F}$ & $\mathbf{2}_{\alpha}^{S}$ & $\{ \pm 3, \pm 1\}$ & $\mathbb{U}$ & $\begin{array}{l}{\left[\Psi, \psi^{\prime}, \Psi^{\prime}, \phi\right]_{-1}} \\
{\left[\Psi, \psi^{\prime}, \Psi^{\prime}, \phi\right]_{1}}\end{array}$ \\
\hline \multicolumn{4}{|c|}{$\left(m_{\nu}\right)_{\alpha \beta} /\langle H\rangle^{3}=-\frac{y_{L_{\alpha i}} y_{i j}^{\prime} y_{j k}^{\prime \prime} y_{k l} y_{R l \beta}}{M_{\psi_{l}}}[$} & \multicolumn{5}{|c|}{$M_{\Psi_{i}} M_{\Psi_{j}^{\prime}} M_{\psi_{k}^{\prime}} I_{4}\left(M_{\phi}, M_{\Psi_{i}}, M_{\psi_{k}^{\prime}}, M_{\Psi_{j}^{\prime}}\right)$} & \\
\hline \multirow{10}{*}{ 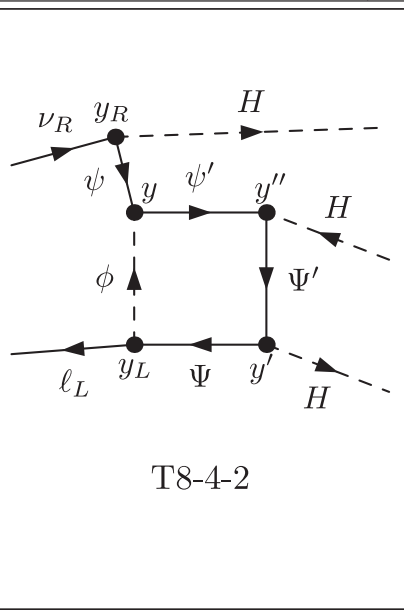 } & & \multirow{2}{*}{$\psi$} & \multirow{2}{*}{$\Psi$} & \multirow{2}{*}{$\psi^{\prime}$} & \multirow{2}{*}{$\Psi^{\prime}$} & \multirow{2}{*}{$\phi$} & & \multirow{2}{*}{ Dark Matter } \\
\hline & & & & & & & \multicolumn{2}{|c|}{ Tree $\quad \mathrm{Ma}$} & \\
\hline & A & $\mathbf{2}_{-1}^{F}$ & $\mathbf{1}_{\alpha}^{F}$ & $\mathbf{1}_{\alpha}^{F}$ & $\mathbf{2}_{\alpha+1}^{F}$ & $\mathbf{2}_{\alpha+1}^{S}$ & $\{-2,0\}$ & $\mathbb{U}$ & $\begin{array}{c}{[\phi]_{-2}^{\diamond}} \\
{\left[\Psi, \psi^{\prime}, \Psi^{\prime}, \phi\right]_{0}^{\diamond}}\end{array}$ \\
\hline & B & $\mathbf{2}_{-1}^{F}$ & $\mathbf{1}_{\alpha}^{F}$ & $\mathbf{3}_{\alpha}^{F}$ & $\mathbf{2}_{\alpha+1}^{F}$ & $\mathbf{2}_{\alpha+1}^{S}$ & $\{ \pm 2,0\}$ & $\mathbb{U}$ & $\begin{array}{c}{[\phi]_{-2}} \\
{\left[\Psi, \psi^{\prime}, \Psi^{\prime}, \phi\right]_{0}}\end{array}$ \\
\hline & \begin{tabular}{|l|}
$\mathrm{C}$ \\
$\mathrm{D}$
\end{tabular} & $\mathbf{2}_{-1}^{F}$ & $2_{\alpha}^{F}$ & $\mathbf{2}_{\alpha}^{F^{\prime}}$ & $\mathbf{1}_{\alpha+1}^{F}$ & $\mathbf{1}_{\alpha+1}^{S}$ & $\{-1\}$ & $\mathbb{U}$ & {$\left[\Psi, \psi^{\prime}, \Psi^{\prime}, \phi\right]_{-1}^{\diamond}$} \\
\hline & \begin{tabular}{|l|}
$\mathrm{D}$ \\
\end{tabular} & $\mathbf{2}_{-1}^{F}$ & $\mathbf{2}_{\alpha}^{F}$ & $\mathbf{2}_{\alpha}^{F}$ & $\mathbf{1}_{\alpha+1}^{F}$ & $3_{\alpha+1}^{S}$ & $\{-3, \pm 1\}$ & $\varnothing$ & {$\left[\Psi, \psi^{\prime}, \Psi^{\prime}, \phi\right]_{-1}^{\diamond}$} \\
\hline & $\mathrm{E}$ & $\mathbf{2}_{-1}^{F}$ & $\mathbf{2}_{\alpha}^{F}$ & $2_{\alpha}^{F^{\prime}}$ & $\mathbf{3}_{\alpha+1}^{F}$ & $\mathbf{1}_{\alpha+1}^{S}$ & $\{-3, \pm 1\}$ & $\varnothing$ & {$\left[\Psi, \psi^{\prime}, \Psi^{\prime}, \phi\right]_{-1}^{\diamond}$} \\
\hline & $\mathrm{F}$ & $\mathbf{2}_{-1}^{F}$ & $\mathbf{2}_{\alpha}^{F}$ & $\mathbf{2}_{\alpha}^{F^{\prime}}$ & $\mathbf{3}_{\alpha+1}^{F}$ & $3_{\alpha+1}^{S}$ & $\{-3, \pm 1\}$ & $\mathbb{U}$ & {$\left[\Psi, \psi^{\prime}, \Psi^{\prime}, \phi\right]_{-1}^{\diamond}$} \\
\hline & $\mathrm{G}$ & $\mathbf{2}_{-1}^{F}$ & $\mathbf{3}_{\alpha}^{F}$ & $\mathbf{1}_{\alpha}^{F}$ & $\mathbf{2}_{\alpha+1}^{F}$ & $\mathbf{2}_{\alpha+1}^{S}$ & $\{ \pm 2,0\}$ & $\mathbb{U}$ & $\begin{array}{c}{[\phi]_{-2}} \\
{\left[\Psi, \psi^{\prime}, \Psi^{\prime}, \phi\right]_{0}}\end{array}$ \\
\hline & $\mathrm{H}$ & $\mathbf{2}_{-1}^{F}$ & $\mathbf{3}_{\alpha}^{F}$ & $\mathbf{3}_{\alpha}^{F}$ & $\mathbf{2}_{\alpha+1}^{F}$ & $\mathbf{2}_{\alpha+1}^{S}$ & $\{ \pm 2,0\}$ & $\mathbb{U}$ & $\begin{array}{c}{[\phi]_{-2}^{\diamond}} \\
{\left[\Psi, \psi^{\prime}, \Psi^{\prime}, \phi\right]_{0}^{\diamond}}\end{array}$ \\
\hline \multicolumn{4}{|c|}{$\left(m_{\nu}\right)_{\alpha \beta} /\langle H\rangle^{3}=-\frac{y_{L_{\alpha} i} y_{i j}^{\prime} y_{j k}^{\prime \prime} y_{k l} y_{R l \beta}}{M_{\psi_{l}}}$} & \multicolumn{5}{|c|}{$\begin{array}{l}{\left[M_{\Psi_{i}} M_{\Psi_{j}^{\prime}} M_{\psi_{k}^{\prime}} I_{4}\left(M_{\phi}, M_{\Psi_{i}}, M_{\psi_{k}^{\prime}}, M_{\Psi_{j}^{\prime}}\right)\right.} \\
\left.J_{4}\left(M_{\phi}, M_{\Psi_{i}}, M_{\psi_{k}^{\prime}}, M_{\Psi_{j}^{\prime}}\right)\right]\end{array}$} & \multirow{3}{*}{ Dark Matter } \\
\hline \multirow{10}{*}{$\underset{\ell_{L}}{y_{L}}$} & & \multirow[t]{2}{*}{$\psi$} & $\Psi$ & $\psi^{\prime}$ & $\Psi^{\prime}$ & $\phi$ & \multicolumn{2}{|c|}{ Excluded $\alpha$} & \\
\hline & & & & & & & Tree & $\mathrm{Ma}$ & \\
\hline & A & $\mathbf{2}_{-1}^{F}$ & $\mathbf{1}_{\alpha}^{F}$ & $\mathbf{1}_{\alpha}^{F}$ & $\mathbf{2}_{\alpha-1}^{F}$ & $\mathbf{2}_{\alpha+1}^{S}$ & $\{-2,0\}$ & $\{-2,0\}$ & $\begin{array}{c}{[\phi]_{-2}^{\diamond}} \\
{\left[\Psi, \psi^{\prime}, \Psi^{\prime}, \phi\right]_{0}^{\diamond}}\end{array}$ \\
\hline & B & $\mathbf{2}_{-1}^{F}$ & $\mathbf{1}_{\alpha}^{F}$ & $\mathbf{3}_{\alpha}^{F}$ & $\mathbf{2}_{\alpha-1}^{F}$ & $\mathbf{2}_{\alpha+1}^{S}$ & $\{ \pm 2,0\}$ & $\{-2,0\}$ & $\begin{array}{c}{[\phi]_{-2}} \\
{\left[\Psi, \psi^{\prime}, \Psi^{\prime}, \phi\right]_{0}}\end{array}$ \\
\hline & $\mathrm{C}$ & $\mathbf{2}_{-1}^{F}$ & $\mathbf{2}_{\alpha}^{F}$ & $2_{\alpha}^{F}$ & $\mathbf{1}_{\alpha-1}^{F}$ & $\mathbf{1}_{\alpha+1}^{S}$ & $\{ \pm 1\}$ & $\{0\}$ & {$[\phi]_{-1}^{\diamond},\left[\Psi, \psi^{\prime}, \Psi^{\prime}\right]_{1}^{\diamond}$} \\
\hline & \begin{tabular}{|l|}
$\mathrm{D}$ \\
\end{tabular} & $\mathbf{2}_{-1}^{F}$ & $2_{\alpha}^{F}$ & $\mathbf{2}_{\alpha}^{F}$ & $\mathbf{1}_{\alpha-1}^{F}$ & $3_{\alpha+1}^{S}$ & $\{-3, \pm 1\}$ & $\varnothing$ & {$[\phi]_{-1}^{\diamond},\left[\Psi, \psi^{\prime}, \Psi^{\prime}\right]_{1}^{\diamond}$} \\
\hline & $\mathrm{E}$ & $\mathbf{2}_{-1}^{F}$ & $\mathbf{2}_{\alpha}^{F^{\prime}}$ & $\mathbf{2}_{\alpha}^{F^{\prime}}$ & $\mathbf{3}_{\alpha-1}^{F^{\prime}}$ & $\mathbf{1}_{\alpha+1}^{S}$ & $\{ \pm 1,3\}$ & $\varnothing$ & {$[\phi]_{-1}^{\diamond},\left[\Psi, \psi^{\prime}, \Psi^{\prime}\right]_{1}^{\diamond}$} \\
\hline & $\mathrm{F}$ & $\mathbf{2}_{-1}^{F}$ & $\mathbf{2}_{\alpha}^{F^{2}}$ & $\mathbf{2}_{\alpha}^{F}$ & $\mathbf{3}_{\alpha-1}^{F}$ & $3_{\alpha+1}^{S}$ & $\{ \pm 3, \pm 1\}$ & $\{0\}$ & {$[\phi]_{-1}^{\diamond},\left[\Psi, \psi^{\prime}, \Psi^{\prime}\right]_{1}^{\diamond}$} \\
\hline & $\mathrm{G}$ & $\mathbf{2}_{-1}^{F}$ & $\mathbf{3}_{\alpha}^{F}$ & $\mathbf{1}_{\alpha}^{F}$ & $\mathbf{2}_{\alpha-1}^{F}$ & $\mathbf{2}_{\alpha+1}^{S}$ & $\{ \pm 2,0\}$ & $\{-2,0\}$ & $\begin{array}{c}{[\phi]_{-2}} \\
{\left[\Psi, \psi^{\prime}, \Psi^{\prime}, \phi\right]_{0}}\end{array}$ \\
\hline & $\mathrm{H}$ & $\mathbf{2}_{-1}^{F}$ & $\mathbf{3}_{\alpha}^{F}$ & $\mathbf{3}_{\alpha}^{F}$ & $\mathbf{2}_{\alpha-1}^{F}$ & $\mathbf{2}_{\alpha+1}^{S}$ & $\{ \pm 2,0\}$ & $\{-2,0\}$ & $\begin{array}{c}{[\phi]_{-2}^{\diamond}} \\
{\left[\Psi, \psi^{\prime}, \Psi^{\prime}, \phi\right]_{0}^{\diamond}}\end{array}$ \\
\hline $\begin{array}{r}\left(m_{\nu}\right)_{\alpha \beta} /\langle H\rangle^{3}= \\
+(M\end{array}$ & & $\begin{array}{r}\frac{y_{i j}^{\prime} y_{j k}^{\prime \prime}}{M_{\psi_{l}}} \\
M_{\Psi_{j}^{\prime}}\end{array}$ & $\begin{array}{l}\frac{y_{k l} y_{R l \beta}}{} \\
+M_{\psi_{k}^{\prime}}\end{array}$ & $\begin{array}{l}{\left[M_{\Psi_{i}} I\right.} \\
J_{4}\end{array}$ & $\begin{array}{l}I_{\Psi_{j}^{\prime}} M_{\psi} \\
M_{\phi}, M\end{array}$ & $\begin{array}{l}I_{k} I_{4}(M \\
\Psi_{i}, M_{\psi_{k}^{\prime}}\end{array}$ & $\begin{array}{l}I_{\phi}, M_{\Psi_{i}}, M_{\psi} \\
\left.\left., M_{\Psi_{j}^{\prime}}\right)\right]\end{array}$ & $\left.M_{\Psi_{j}^{\prime}}\right)$ & \\
\hline
\end{tabular}




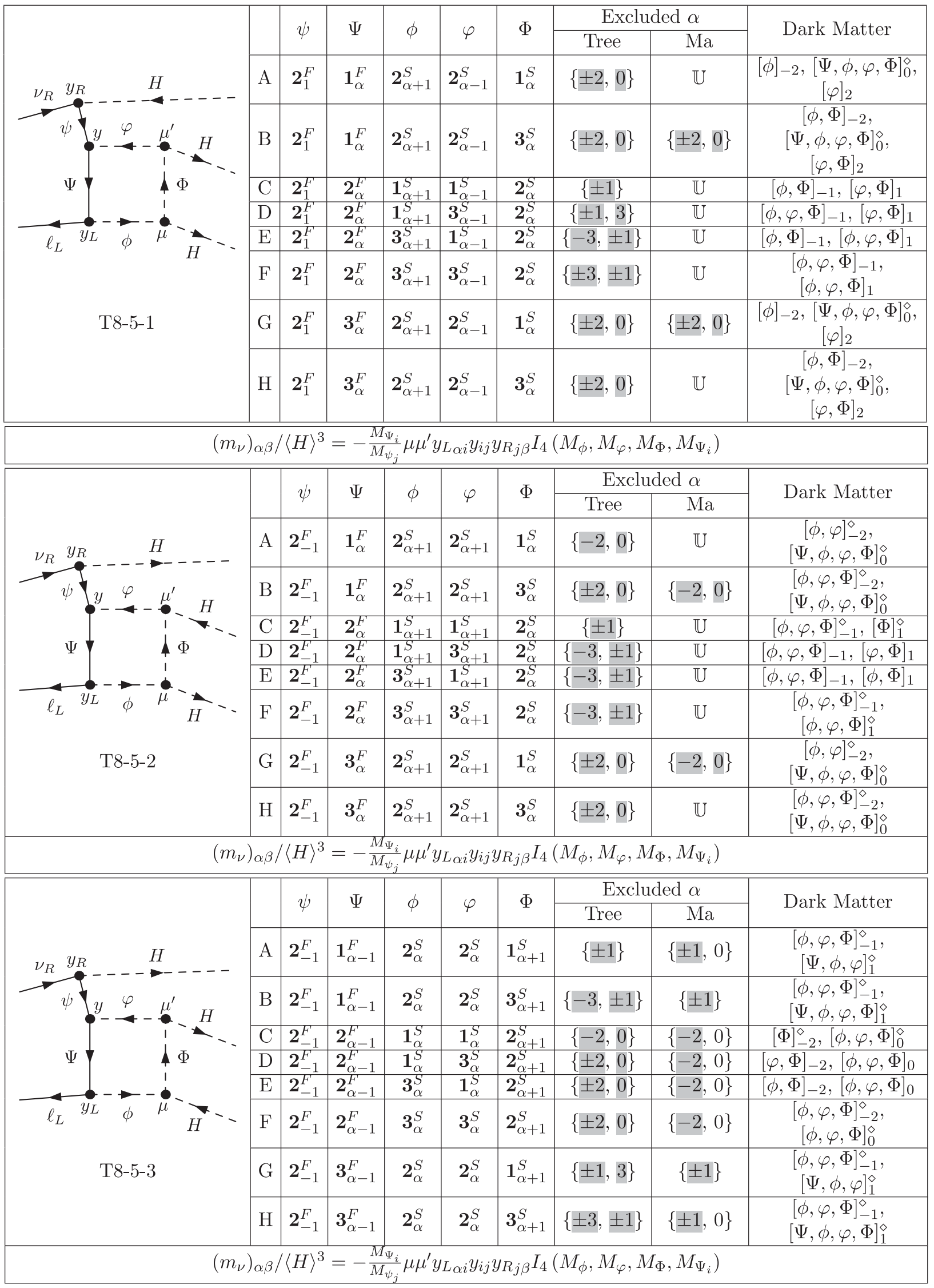


external fields (Higgs VEV $, \nu_{L}, \nu_{R}$ ). This appears in both tree level and loop level diagrams, we must discard such models in our original paper. This will affect the numbers of the genuine models and the excluded values of $\alpha$ in some tables, but the conclusions of the article would not change. We listed the necessary modifications one by one in the following.

(i) the model A of diagram F2-2-3 in Table I should be removed, and then the model B should be named as A.

(ii) line 15-16 of the left column on page 4, should read " ... There are only five such diagrams T2-2, T3-6, T8-3, T8-4, and T8-5, as shown in Fig. 2.".

(iii) line 18-19 of the left column on page 4, should read "... shows other finite diagrams for which either the tree level contributions or the Ma diagrams cannot be forbidden ...".

(iv) line 23-24 of the left column on page 4, should read "... Eventually we find only 14 irreducible Feynman diagrams ...".

(v) diagram T6-4 in Fig. 2 should be removed, and added to Fig. 6.

(vi) the words "T3-6-1-A" throughout the paper should read "T3-6-2".

(vii) line 26 of the right column on page 11 , should read "... and there are only 12 models which contain ...".

(viii) the first row of Table $\mathrm{V}$ should be removed.

(ix) line 19 of the left column on page 13, should read "... We find 14 one-loop diagrams in ...".

(x) line 1 of the right column on page 17, should read “... are generated from the topologies $\mathrm{T} 2$, T3, and T8 ...".

(xi) in Table VI, for the diagram T2-2-1, B and D models should be removed, and the names A,C,E,F should be replaced by A,B,C and D respectively. For model A of diagram T2-2-4, the excluded values of $\alpha$ in the "Tree" column should read $\{ \pm 1\}$.

(xii) in Table VII, the whole results for diagram T3-6-1 should be removed, and T3-6-2 should be named as T3-6-1.

(xiii) Table VIII should be removed.

(xiv) Table IX in the original paper should be replaced by Table VIII here and renamed as Table VIII. The text "Table VIIX" throughout the paper should read "Table VI-VIII". 